\title{
DQPSK: When Is a Narrow Filter Receiver Good Enough?
}

\author{
Francesco Vacondio, Amirhossein Ghazisaeidi, Alberto Bononi, Member, IEEE, and \\ Leslie A. Rusch, Senior Member, IEEE
}

\begin{abstract}
In this paper, we investigate experimentally and via simulation the pros and cons of a narrow filter receiver for differential quadrature phase-shift keying based on a single optical filter and eschewing the conventional asymmetrical Mach-Zehnder interferometer structure. We quantify the performance differences between the two receivers, allowing system designers and operators to determine when the less complex narrow filter receiver might be the appropriate choice. We numerically optimize the 3-dB bandwidth and center frequency of the narrow filter and show it is more robust to carrier frequency detuning than the conventional solution. We show that the narrow filter receiver is more tolerant to chromatic dispersion (CD) than the conventional one, and equally tolerant to first-order polarization-mode dispersion. We show the impact of the 3-dB bandwidth on the receiver performance when CD accumulates. Finally, we show via experiments and simulations that the $3 \mathrm{~dB}$ advantage of the conventional receiver vanishes when the nonlinear impairments are fiber nonlinearities; comparing the two receivers at the optimum launch power for a $25 \times 80 \mathrm{~km}$ system, the difference in optical SNR margin is reduced to $\sim 1.6 \mathrm{~dB}$. Experiments are conducted at $42 \mathrm{~Gb} / \mathrm{s}$ using a commercially available narrow filter for reception.
\end{abstract}

Index Terms-Differential phase-shift keying, modeling, optical fiber communication, optical receivers.

\section{INTRODUCTION}

$\mathbf{P}$ HASE modulation has drawn much attention in the last few years for next-generation spectrally efficient optical networks [1]. In particular, optical differential quadrature phase-shift keying (DQPSK) is emerging as a promising solution, and the technology is today mature enough to permit validations outside the research labs [2]. The conventional receiver for DQPSK, depicted in Fig. 1(a), is composed of an optical filter, two asymmetrical Mach-Zehnder interferometer (AMZI) structures, and two balanced photodiodes. Its complexity is twice the complexity of a binary DPSK (DBPSK) receiver. In order to increase the cost/benefit ratio of phase-modulated formats and to eventually avoid the interferometric structure, much effort has been focused in proposing

Manuscript received January 30, 2009; revised June 16, 2009 and July 29, 2009. First published August 07, 2009; current version published September 25 , 2009. This work was supported in part by 2007-2009 Québec-Italy Executive Program for Scientific Development, Project 13.

F. Vacondio, A. Ghazisaeidi, and L. A. Rusch are with the Center of Optics, Photonics and Laser (COPL) and with the Department of Electrical and Computer Engineering, Université Laval, Québec, QC G1V0A6, Canada (e-mail: francesco.vacondio.1@ulaval.ca).

A. Bononi is with Dipartimento di Ingegneria dell'Informazione, Università degli Studi di Parma, Parma 43100, Italy.

Color versions of one or more of the figures in this paper are available online at http://ieeexplore.ieee.org.

Digital Object Identifier 10.1109/JLT.2009.2029538
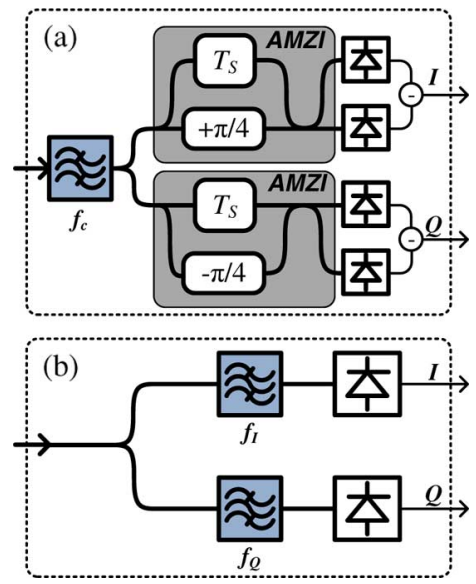

Fig. 1. (a) Conventional receiver and (b) narrow filter receiver for DQPSK signals. $T_{s}$ is a symbol time, $f_{c}$ is the carrier frequency, $f_{I}=f_{c}+\delta f$, and $f_{Q}=f_{c}-\delta f$.

alternative, lower complexity receivers for both binary and quadrature phase-shift keying.

One such reduced complexity receiver [3], [4] is based on polarization, where the two arms of the AMZI are replaced by the slow and the fast axis of a polarization maintaining fiber, whose differential group delay (DGD) equals one symbol interval; the signals out of the two axes are then mixed before photodetection. This receiver is extended to DQPSK in [5], demonstrating experimentally the main advantage of such a receiver, which is the relative ease of implementation and the wide range over which the DGD can be tuned, hence permitting the use of the same receiver for different bit rates. The main drawback is that the signal polarization needs to be controlled with very high precision and stability, thus increasing cost and complexity.

We turn our attention to two other promising receivers that use a single photodetector for DBPSK; in the case of DQPSK, these receivers have one photodetector in each of the I and Q arms. In the first receiver, the AMZI structure is maintained as in Fig. 1(a); however, only one port (either the constructive or the destructive port) is populated with a photo detector [6]. In the second receiver, the AMZI structure is not used and instead only a narrowband filter is found in each branch, as illustrated in Fig. 1(b) [7]; the narrowband filters replace the channel select filter and the AMZI structure. It is well known that the conventional receiver for DQPSK has cosine-shaped equivalent transfer functions in the frequency domain. They are plotted in Fig. 2 versus the frequency normalized to the symbol rate $R$. The frequency responses are periodic and shifted with respect to the carrier frequency. Thick lines indicate the main lobes of 


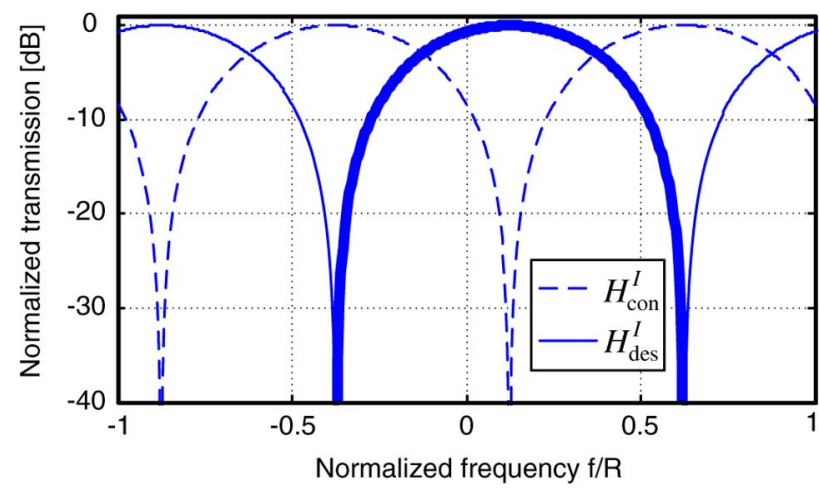

(a)

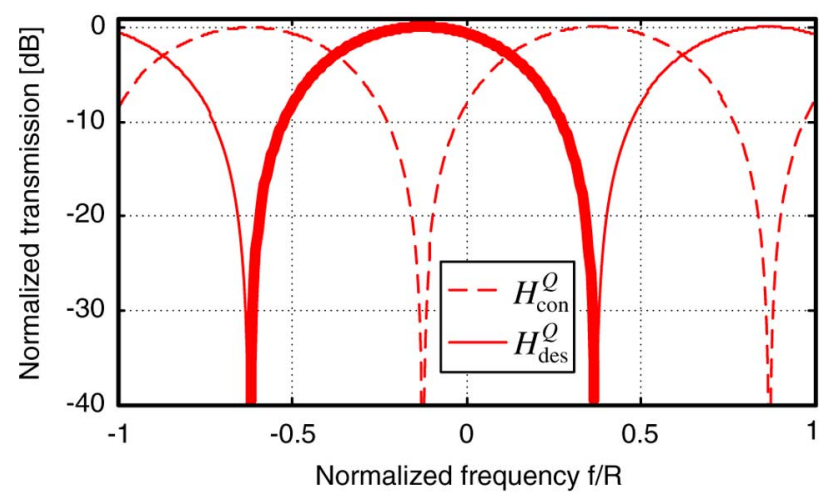

(b)

Fig. 2. Four equivalent periodic filters of the DQPSK receiver. Dashed lines for constructive ports and solid lines for destructive ports. Thick lines represent the lobes that the narrow filter receiver approximates: (a) for the in-phase component and (b) for the quadrature component.

the destructive ports in both the I and Q cases. The narrowband filter receiver seeks to approximate these main lobes for each branch; the main lobes are centered at $+1 / 8$ units of normalized frequency for the in-phase component and $-1 / 8$ units for the quadrature component.

Researchers have examined the DBPSK version (single branch) of the narrow filter receiver in Fig. 1(b), [8]-[12], with only very recent results for the DQPSK version [13]. Since the narrow filter receiver (without AMZI) approximates the single-ended AMZI with only one photodiode, we expect them to show similar performance. We confine our attention thus to the narrow filter receiver and compare it to the conventional AMZI balanced receiver of Fig. 1(a).

We demonstrate via bit error rate (BER) measurements and simulations that the narrow filter receiver has a $3 \mathrm{~dB}$ penalty in the linear, dispersion-free regime, compared with the conventional AMZI balanced receiver. By avoiding the interferometric structure and using a single-ended photodiode instead of a balanced one, the narrow filter receiver enjoys reduced cost and complexity, as well as greater flexibility. We will show that the tolerance to nonoptimal tuning of its center frequency is greater than the tolerance of the conventional receiver to frequency detuning. We compare quantitatively the performance of the narrow filter to that of the conventional receiver. In this paper, we present a complete analysis of advantages and drawbacks of the narrow filter receiver, assessing its performance in simulations and experiments vis-à-vis linear and nonlinear transmission impairments.

The narrow filter receiver is by construction spectrally efficient; channels in a WDM system can be packed more closely than when using the wider band, conventional AMZI receiver of Fig. 1. Typically when packing channels more tightly to improve spectral efficiency, we expect the narrower channel select filters to induce greater intersymbol interference (ISI). When using an AMZI receiver, we can tweak the free spectral range (FSR) such that the overall frequency response ${ }^{1}$ tends to equalize the line, to combat ISI. The spectral efficiency of a narrow filter WDM system can be further increased using the same techniques used with the AMZI receiver. The narrow filter receiver is simply chosen to match the main lobe of the AMZI destructive port when using the most advantageous FSR. Note that for both binary and quadrature DPSK, narrowing the channel selective filter makes the conventional AMZI receiver more resilient to accumulated chromatic dispersion (CD), as well as more spectrally efficient [14]-[18]. It is not surprising then that we find the narrow filter receiver exhibits the same greater tolerance to ISI as the conventional AMZI with partial D(Q)PSK.

For DBPSK it has already been reported that, in the nonlinear regime, the performance of the conventional receiver tends to that of the single-ended receiver (where the balanced receiver is replaced with a single-ended photodiode on one of the two output arms of the AMZI). The advantage of balanced reception disappears as the dominant noise is nonlinear phase noise (NLPN) and not amplitude noise [19], [20]. Since the narrow filter receiver emulates the single-ended one, it is expected that under nonlinear propagation in fiber its performance should be equivalent to those of the conventional receiver. We will show that this is the case.

The paper is organized as follows. Section 2 is devoted to the comparative performance analysis, both experimental and numerical, of the two receivers in back-to-back operation, and in the presence of $\mathrm{CD}$ and first-order polarization-mode dispersion (PMD). In Section 3, we present measurements and simulations of the behavior of the two receivers in the nonlinear regime. Finally, conclusions are drawn in Section 4.

\section{LINEAR IMPAIRMENTS}

\section{A. Experimental Setup}

The experimental setup for the investigation of linear impairments is sketched in Fig. 3. A polarization-controlled tunable laser is modulated with a commercial I/Q modulator (SHF 46213). The modulator is composed of two Mach-Zehnder modulators (MZM) nested in an interferometric structure [21].

We use the data and data bar (logical inverse) outputs of a $R=21 \mathrm{~Gb} / \mathrm{s}$ pattern generator for our I and $\mathrm{Q}$ components. Two challenges are imposed. First, we must delay the two data outputs to decorrelate them so that the overall data rate is 42 $\mathrm{Gb} / \mathrm{s}$ (that is, to achieve two independent data streams). However, the delay must be a multiple of the bit duration in order to successfully demodulate the streams (our phase-matched RF cables introduced a relative delay of precisely 21 information

\footnotetext{
${ }^{1}$ The overall frequency response is determined by both the channel selection optical filter and the AMZI structure, as well as the photo-detection process.
} 


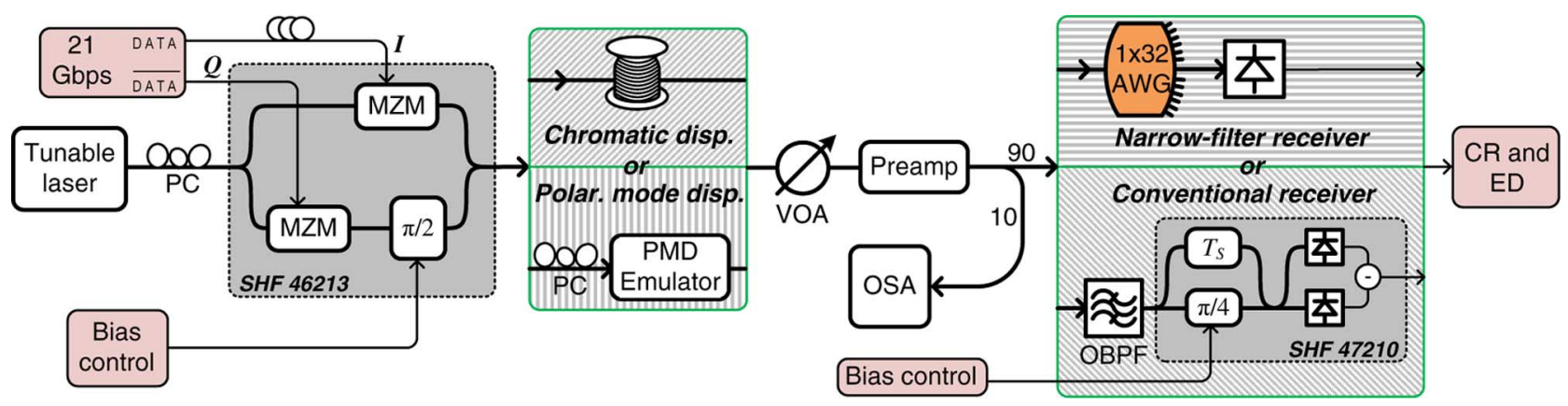

Fig. 3. Experimental setup for the investigation of $\mathrm{CD}$ and first-order PMD tolerance. PC: polarization controller, MZM: Mach-Zehnder modulator, PMD: polarization-mode dispersion, VOA: variable optical attenuator, EDFA: erbium-doped fiber amplifier, OSA: optical spectrum analyzer, AWG: arrayed waveguide grating, OBPF: optical bandpass filter, CR: clock recovery, ED: error detector.

bits). The $\pi / 2$ phase shift is manually adjusted and constantly monitored.

Two transmission media are considered. To investigate the effects of $\mathrm{CD}$, the signal enters a spool of optical fiber. To investigate the effects of PMD, the signal passes through a PMD emulator. The emulator consists of a polarization beam splitter (PBS) to split the signal into a slow and a fast component. The slow component is delayed by an optical delay line and recombined with the fast component by means of a second PBS. A polarization controller in front of the PMD emulator is set to assure that the signal is aligned at $45^{\circ}$ with the first PBS in the PMD emulator.

At the receiver side, the signal power is controlled with a variable optical attenuator (VOA). A preamplifier stage (composed of an erbium-doped fiber amplifier (EDFA), a $1.4 \mathrm{~nm}$ optical bandpass filter, and a second EDFA) sets the SNR. The spectrum of the noisy signal is constantly monitored with an optical spectrum analyzer (OSA) via the $10 / 90$ power splitter. The signal can be received by either of the two available receivers, narrow filter or conventional. The narrow filter receiver is implemented with one channel of a commercially available $1 \times 32$ arrayed waveguide grating (AWG); the measured AWG spectrum is shown in Fig. 4 along with the theoretical AMZI spectrum and a Gaussian spectrum with $3-\mathrm{dB}$ bandwidth equal to $0.6 R$. As we can see, our narrow filter closely approximates the Gaussian shape. The photodiode incorporates a transimpedance amplifier. The conventional receiver is formed by a channel select filter followed by a commercial optical DPSK demodulator. The channel is selected by an optical bandpass filter (OBPF) with a 3-dB bandwidth of $\sim 50 \mathrm{GHz}$. The balanced photoreceiver also incorporates a transimpedance amplifier.

Following photodetection, the clock is reconstructed with a commercial clock recovery circuit and fed to a BER tester together with the received data. Note that no differential encoder (at the transmitter) or differential decoder (at the receiver) is used, so the received pattern is different from the transmitted pattern. For experimental convenience, we chose a $2^{7}$ DeBrujin sequence at the transmitter and programmed the correct differentially decoded patterns into the BER tester [21]. The I and Q streams were accessed sequentially using, in the case of the conventional receiver, a commercial (SHF model number) DBPSK demodulator or, in the case of the narrow filter receiver, by manually tuning the central frequency of the AWG.

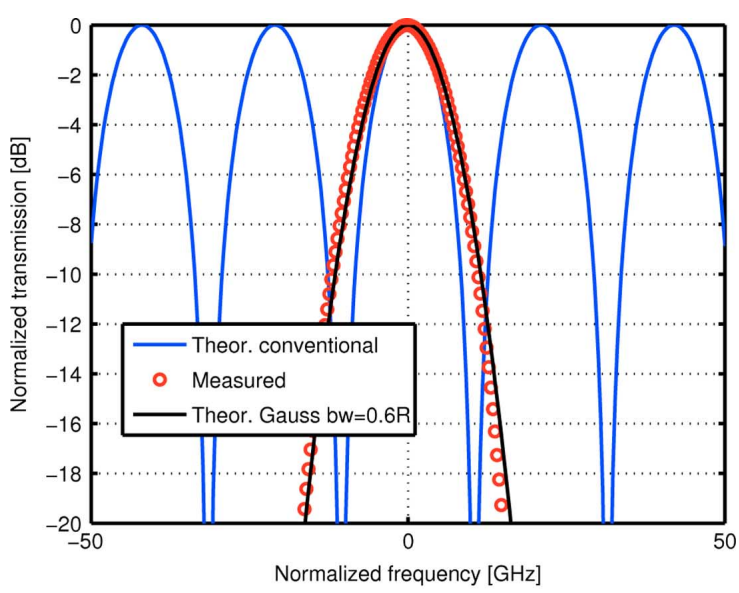

(a)

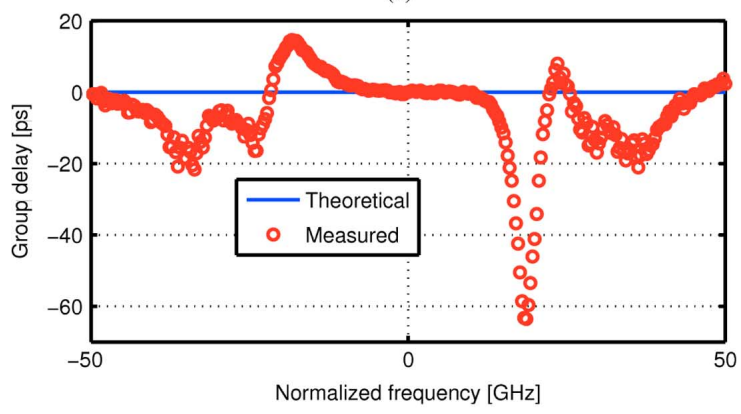

(b)

Fig. 4. Measured transmission and group delay profiles of the narrow filter employed in the experiments. The theoretical shapes are also reported along with the transmission spectrum of a Gaussian filter with 3 -dB bandwidth equal to $0.6 R$, where $R$ is the symbol rate. (a) Intensity profile; (b) Group delay profile.

\section{B. Numerical Model}

In addition to the experimental investigation of the performance of the narrow filter and the conventional receivers, we also examined their performance via numerical simulation. We model the optical source as an ideal laser. The MZM is modeled as described in [22], [23], where the output field of a single MZM is

$$
E_{\text {out }}=\frac{E_{\text {in }}}{2}\left(e^{j \phi_{1}}+\gamma e^{j \phi_{2}}\right)
$$

where $E_{\text {in }}$ is the input field to the MZM, $\phi_{1}$ and $\phi_{2}$ are the phase shifts in the two Mach-Zehnder arms, and $\gamma$ is related 


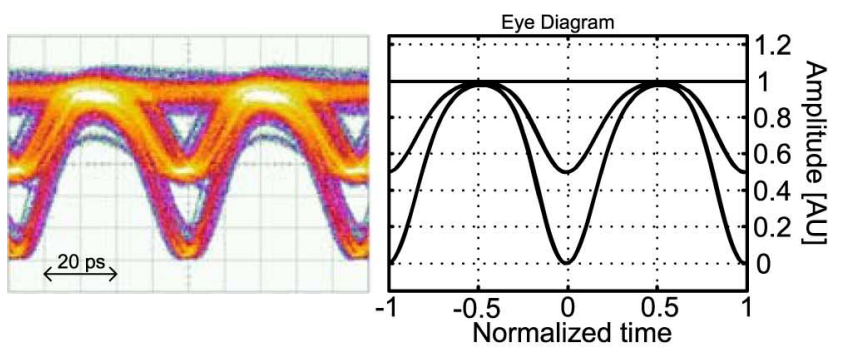

(a)

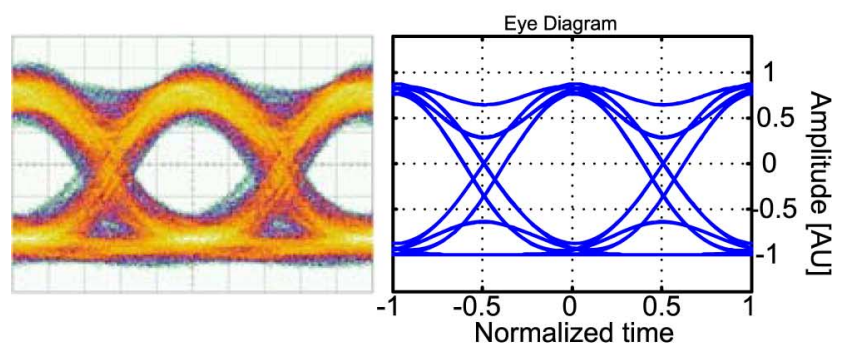

(b)

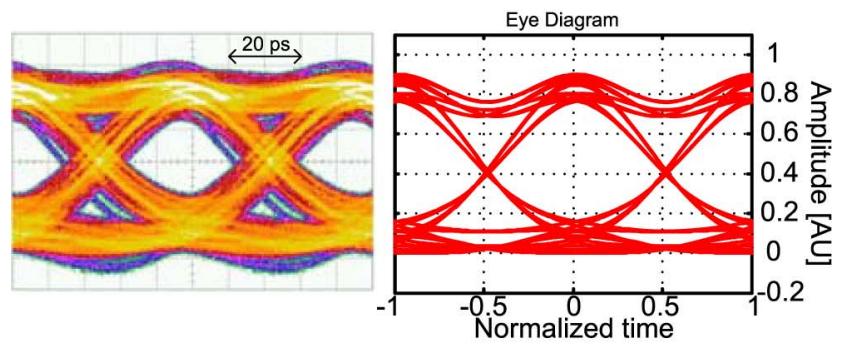

(c)

Fig. 5. Measured (left column) and simulated (right column) eye diagrams. (a) DQPSK optical waveform; (b) Conventional receiver; (c) Narrow filter receiver.

to the DC extinction ratio $\epsilon$ per $\gamma=(\sqrt{\epsilon}-1) /(\sqrt{\epsilon}+1)$. The pattern generator is modeled as an ideal signal source filtered with a fifth-order low-pass Bessel filter. The 3-dB bandwidth of this filter and the extinction ratio of the MZM are chosen to best fit experimental results. The measured frequency responses are used for the optical filters in the simulation. Photodetection is supposed ideal and another fifth-order low-pass Bessel filter models the finite electric bandwidth of the receiver (3-dB bandwidth is again chosen to best fit the experimental back-to-back results). Fig. 5 shows the measured and simulated eye diagrams of the optical DQPSK signal and of the electrical signals output from the two receivers.

The bit patterns in the simulations are pseudorandom quaternary sequences [24]. The BER is simulated with a semianalytical approach accounting for the memory of the system as well as optical and electrical nonideal filter shapes. Several such approaches exist [25]-[30], and all share the same line of thought. The received signal is written as $r(t)=s(t)+n(t)$, i.e., the sum of the useful signal and a noise term (amplified spontaneous emission (ASE) from amplifiers). Noise $n(t)$ is modeled as a Gaussian (possibly colored) random process. The signal $s(t)$ is expanded in a suitable basis. We use the Karhunen-Loève basis to facilitate the inclusion of nonideal pre- and postreception filters. The moment-generating function (MGF) of the decision variable (a quadratic form of the sum of possibly correlated

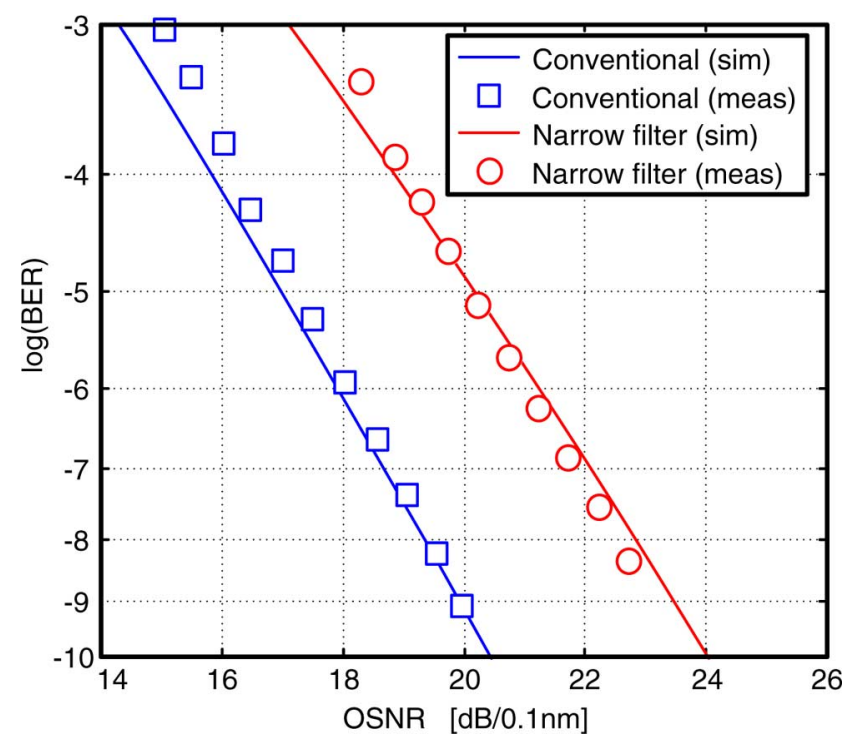

Fig. 6. Experimental and simulated back-to-back BER for both receivers under investigation. Symbol rate is $21 \mathrm{Gbaud}$, bit rate is $42 \mathrm{~Gb} / \mathrm{s}$.

Gaussian random variables) can then be derived analytically. Finally, the probability density function, and hence the BER, is obtained as the inverse Laplace transform of the MGF. A practical numerical procedure for such an inversion is the so-called saddle point integration. Details of the algorithm we used can be found in [30].

\section{Back-to-Back Operation}

Fig. 6 shows the back-to-back BER measurements along with simulation results. Simulated performance very closely matches measured performance; in particular, a penalty of $\sim 3.2 \mathrm{~dB}$ for the narrow filter is correctly predicted by our simulator. Such a penalty is in agreement with both the simulation results of [13] and the intuition that the narrow filter emulates the single-ended DQPSK receiver. The novelty in our work is threefold: 1) we give experimental validation of BER (not only eye diagrams); 2) we provide simulation results that are both more accurate (using realistic filter models) and more precise as we investigate also the low BER regime, including good agreement with the experimental data; and 3) we use a realistic network element (AWG) as an optical filter, instead of the monochromator stage of an OSA as in [13].

We next numerically investigate performance dependence on filter center frequency and 3-dB bandwidth. We vary both parameters over a wide range of values and measure the optical SNR (OSNR) penalty at BER $=10^{-5}$. Contour plots in Fig. 7(a) give results for the quadrature component (the center frequency is negative) and Fig. 7(b) the in-phase component (the center frequency is positive). Frequencies in the contour plots are normalized to the bit rate; stars indicate the minimal penalty (optimal parameters). As in [13], the optimal center frequency does not correspond to $R / 8$ but to a slightly higher value. We find the optimal center frequency to be close to $0.18 R$, for a $3-\mathrm{dB}$ bandwidth of $0.6 R$. In [13], an optimal center frequency of $0.173 R$ was reported. The $3-\mathrm{dB}$ bandwidth was not swept in [13], which might explain the small 


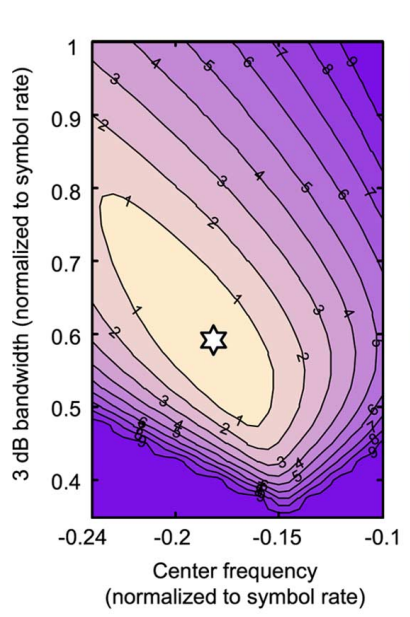

(a)
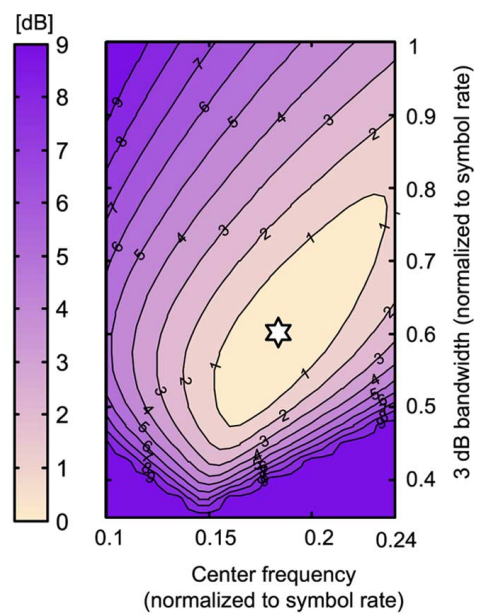

(b)
Fig. 7. OSNR penalty at $B E R=10^{-5}$ for the narrow filter receiver as a function of filter 3-dB bandwidth and filter center frequency for (a) the quadrature component and (b) the in-phase component. Stars indicate minimal penalty.

discrepancy. The bowl around the minimal OSNR penalty is quite shallow for the narrow filter receiver: the OSNR penalty is confined to less than $1 \mathrm{~dB}(2 \mathrm{~dB})$ for a range of more than $0.15 R(0.25 R)$ around the optimal value. At $21 \mathrm{Gbps}$, this corresponds to a $1 \mathrm{~dB}$ tolerance of $\pm 1.57 \mathrm{GHz}$ ( $2 \mathrm{~dB}$ tolerance of $\pm 2.62 \mathrm{GHz}$ ).

A major concern in direct detection DQPSK systems is the robustness to frequency offset (or detuning) between the carrier and the receiver [31]-[33]. To explore tolerance to detuning for both receivers, we introduce $\delta \phi$, the error in-phase adjustment of the receiver. For instance, the phase shift of the in-phase arm would be $\pi / 4+\delta \phi$. Such an error induces a frequency detuning of $\Delta f=\delta \phi R /(4 \pi)$. In the case of the narrow filter receiver, the detuning is simply a mismatch between the filter center frequency and the carrier frequency. We investigate numerically the tolerance of the receivers to this effect. Fig. 8 shows the results in terms of OSNR penalties at BER $=10^{-5}$. The penalties are referred to the performance of each receiver when $\Delta f=0$. We can see that the narrow filter receiver is more tolerant (by slightly more than a factor 2) to frequency detuning.

In the next sections, we examine the impact of linear fiber impairments (CD and PMD) via experiment and simulation.

\section{Chromatic Dispersion}

Simulations of BER-equivalent $Q$-factor for DQPSK [13] found the narrow filter receiver has a higher tolerance to accumulated CD by a factor of $\sim 2$. We extend that work by simulating OSNR penalties. Two experimental measurements were captured and found to coincide with simulation results. We also numerically investigate the influence of the filter 3-dB bandwidth on the system performance in the presence of $\mathrm{CD}$. While OSNR penalties are more difficult to calculate, ${ }^{2}$ they provide a very useful performance metric for the network engineer. Experimentally, we use one of two spools of single-mode

${ }^{2}$ We calculate BER down to $10^{-5}$ versus OSNR for each CD value; note that [13] fixed the OSNR to a regime where BER was assured to be above $10^{-4}$ and only one BER point was found per CD value.

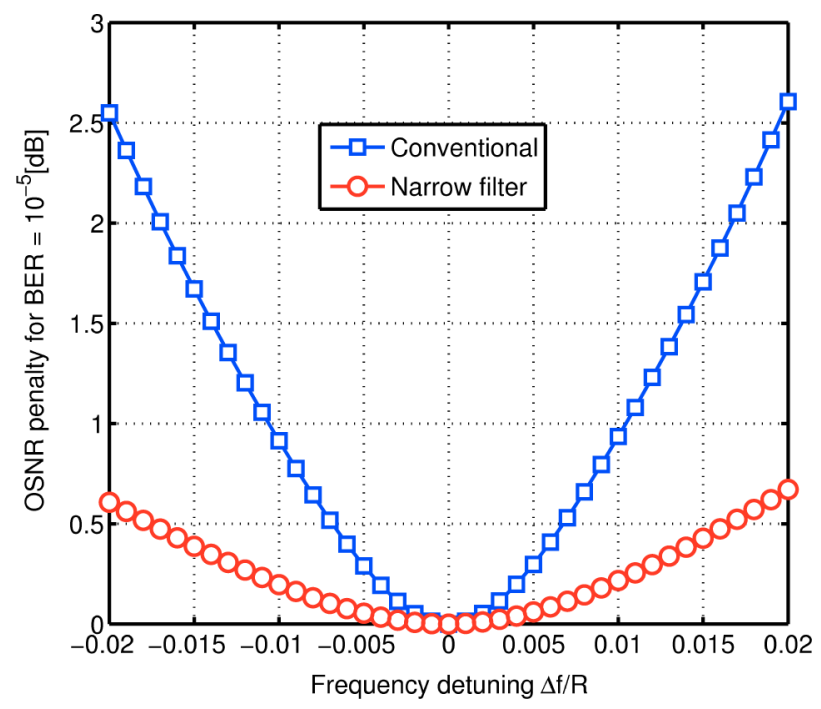

Fig. 8. OSNR penalty for a $B E R=10^{-5}$ for the narrow filter receiver and for the conventional receiver as a function of the detuning frequency.

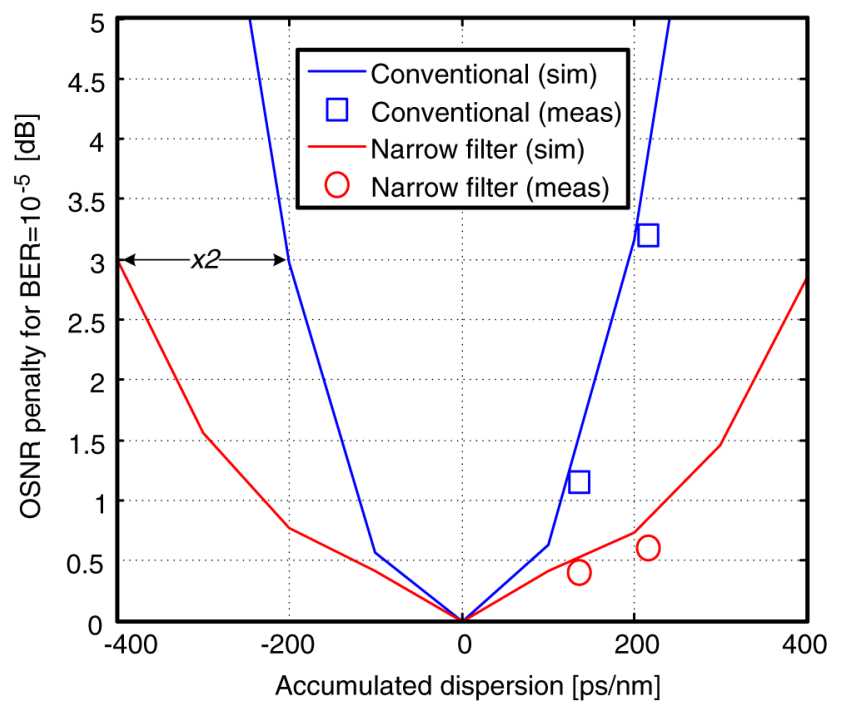

Fig. 9. Simulated OSNR penalties at $B E R=10^{-5}$ as a function of accumulated $\mathrm{CD}$ for both receivers under investigation. Two experimental points are also provided for comparison.

fiber (SMF) (136 and $216 \mathrm{ps} / \mathrm{nm}$ of accumulated dispersion, respectively). Input power to the fiber is $-3 \mathrm{dBm}$, ensuring linear propagation. Simulations are also carried out in this linear regime; the fiber is modeled as a simple all-pass filter. We use as a reference point the OSNR required to achieve $\mathrm{BER}=10^{-5}$ when no dispersion is present (back-to-back). We report the OSNR penalty at BER $=10^{-5}$ as a function of the accumulated dispersion. Please note that throughout the paper (unless otherwise specified) the OSNR is referred to a standard bandwidth of $0.1 \mathrm{~nm}$.

$\mathrm{CD}$ results are summarized in Fig. 9. The improved resilience of the narrow filter receiver to accumulated $\mathrm{CD}$ is clearly visible. The results of two measurements (at 136 and $216 \mathrm{ps} / \mathrm{nm}$ of accumulated dispersion) can be seen to fall very close to the simulated data. The narrow filter receiver shows a factor of $\sim 2$ improvement, i.e., the same OSNR penalty is reached by accumulating about twice the total CD. 


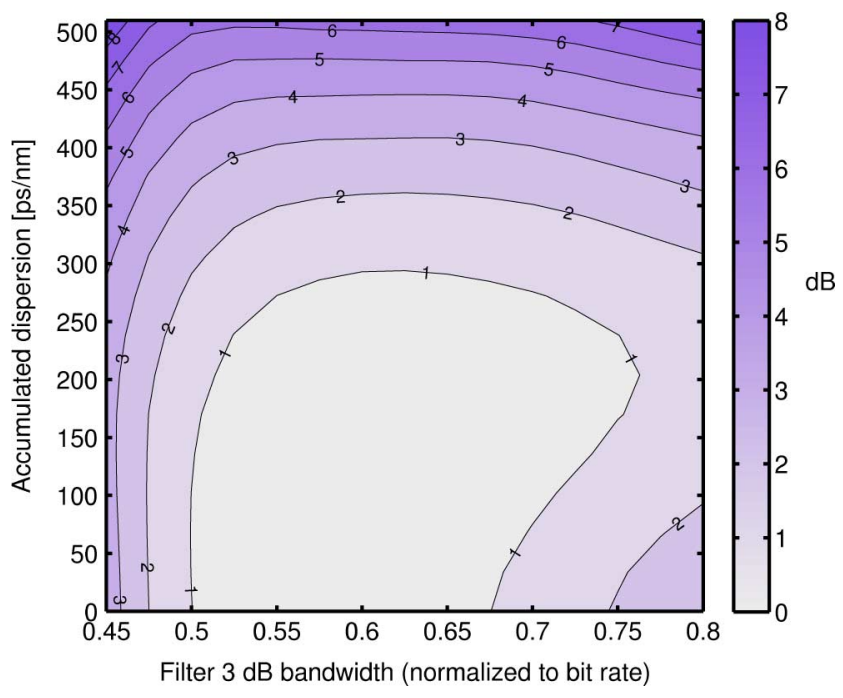

Fig. 10. Simulated OSNR penalties at $\mathrm{BER}=10^{-5}$ as a function of accumulated $\mathrm{CD}$ and filter bandwidth. The optimal value of the $3 \mathrm{~dB}$ optimal bandwidth only slightly depends on accumulated dispersion.

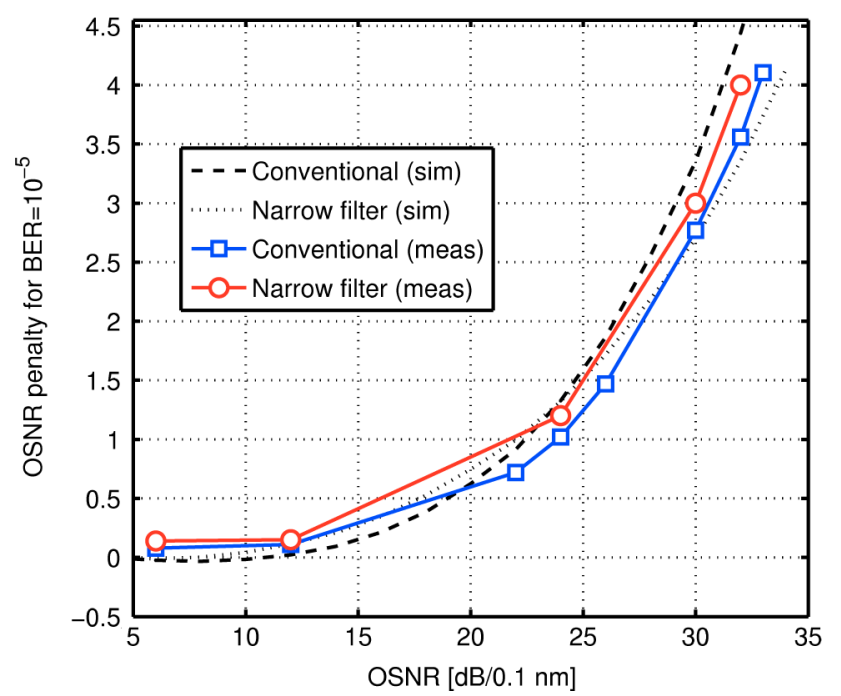

Fig. 11. Experimental OSNR penalties at a BER $=10^{-5}$ due to first-order PMD as a function of DGD for both receivers under investigation, for the worst case 50/50 power split.

\section{E. Polarization-Mode Dispersion}

Previous results on PMD and DQPSK signals focused on the performance of the AMZI receiver as a function of DGD [34]. We report the performance of the narrow filter receiver, in particular as it compares to the performance of the conventional AMZI receiver, when first-order PMD is present leading to DGD. We vary the amount of DGD via the PMD emulator with the input state of polarization giving a 50/50 power split on the two principal states of polarization, and we again record the OSNR penalty at BER $=10^{-5}$ for the two receivers. Results are summarized in Fig. 11. There is a negligible difference in the penalties; hence, the two receivers have the same tolerance to first-order PMD. We also show, in dashed and dotted lines, the results of simulations of first-order PMD for the two receivers. Results are in good qualitative agreement with the measured values.

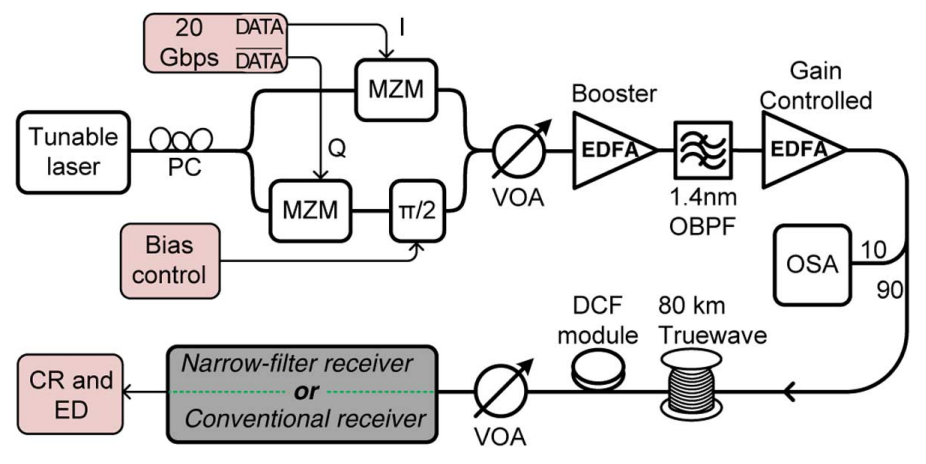

Fig. 12. Experimental setup for the nonlinear transmission experiment.

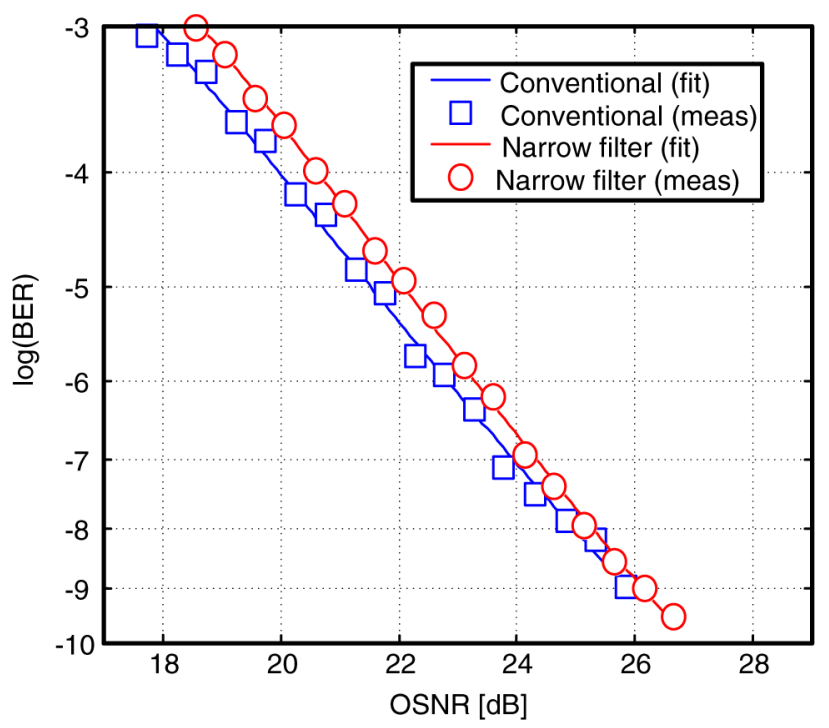

Fig. 13. BER for both receivers in the nonlinear regime.

\section{NONLINEAR PHASE NOISE}

In this section, we evaluate the tolerance of the narrow filter receiver to fiber nonlinearities, i.e., intrachannel effects and NLPN. NLPN arises from amplifier ASE that is translated to the phase of the signal by self-phase modulation in fiber [35].

\section{A. Experimental Results and Discussion}

The experimental setup is depicted in Fig. 12. After the DQPSK modulator, a first VOA controls the power of the useful signal. An amplification stage built with a booster EDFA, a 1.4 nm optical filter, and a gain-controlled EDFA provide sufficient power and set the OSNR. With a 10/90 splitter, the OSNR is monitored after the transmitter. A signal power of $\sim+13 \mathrm{dBm}$ is injected into the fiber, in order to trigger nonlinearities. The DQPSK signal and the noise interact when traveling in a spool of $80 \mathrm{~km}$ of Truewave (TW) fiber, and a matched DCF module provides full compensation of the CD. A second VOA controls the power on the receiver to keep it constant. The narrow filter receiver and the conventional receiver are used in turn, and the BER is measured as per the previous section. The results are presented in Fig. 13. As we expected, the performance of the receivers now overlap: the $3 \mathrm{~dB}$ advantage of the conventional receiver has vanished. A similar result, but for binary DPSK, was shown in [19], where the single-ended receiver and the 


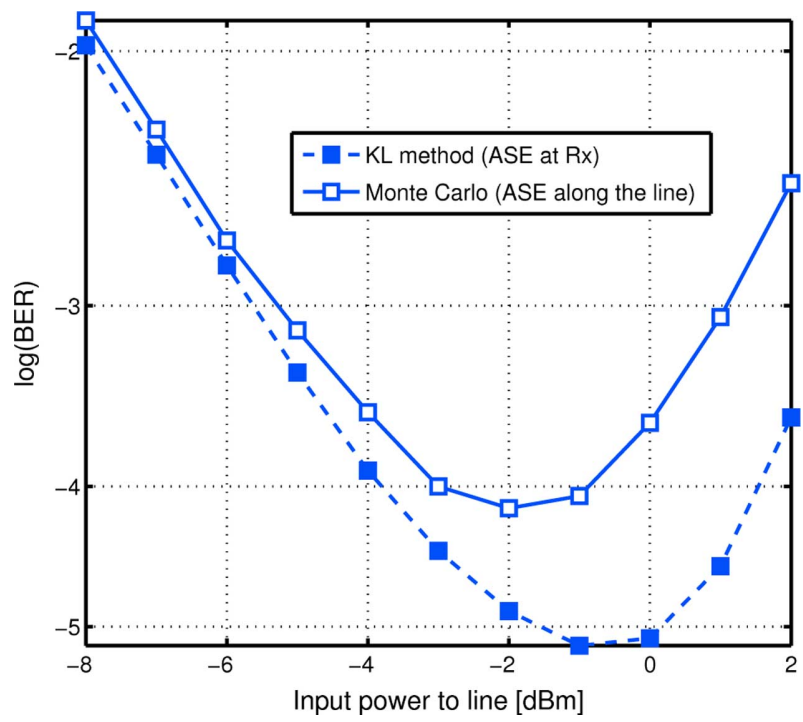

Fig. 14. BER when KL method is used or MC method is used. Fully compensated DQPSK 21 Gbaud single-channel $25 \times 80 \mathrm{~km}$ TW system, conventional receiver only.

conventional receiver were shown to have the same BER in the nonlinear regime, notwithstanding the use of a simple photodiode instead of the balanced one. We demonstrate here for the first time that for DQPSK the narrow filter receiver also has the same performance as the conventional one, obviating not only the balanced photodetection but also the AMZI itself.

\section{B. Numerical Model}

The KLSE method with white ASE noise used in the previous section accounts for intrachannel nonlinearities, but not for NLPN, since the nonlinear signal-noise interaction alters the statistics of the received ASE in a complex way. To appreciate the impact of NLPN in a realistic disperion-managed (DM) scenario, we simulated a 21 Gbaud DQPSK single-channel $25 \times 80$ $\mathrm{km}$ DM line with TW transmission fiber (dispersion coefficient $D=8 \mathrm{ps} / \mathrm{nm} / \mathrm{km}$, nonlinear coefficient $\gamma=1.7 \mathrm{~W} / \mathrm{km}$ ) with full in-line compensation. No pre- and postcompensation was added, and the EDFA noise figure was $F=6 \mathrm{~dB}$. In Fig. 14, we show the BER versus input power for the conventional receiver, calculated with both the KLSE method and with brute-force, error-counting Monte Carlo (MC) estimation with at least 100 errors collected for each point [36].

Both KLSE and MC use the split-step Fourier method [37] for field propagation, but in the MC case the noise is added along the line and propagated with the signal, thus making simulations much longer. We note that KLSE estimation becomes overly optimistic at powers larger than $-5 \mathrm{dBm}$, since NLPN is becoming the dominant impairment. Having established the significance of NLPN contribution to the fiber nonlinearity for the scenario under investigation, we will thus use only $\mathrm{MC}$ estimation to assess the relative performance of the two receivers of Fig. 1 in the nonlinear regime. In order to do so, we simulated the same DM line as before, but with either TW or SMF $(D=17 \mathrm{ps} / \mathrm{nm} / \mathrm{km}$, nonlinear coefficient $\gamma=1.3 \mathrm{~W} / \mathrm{km}$ ) transmission fiber. The comparison of the two receivers is provided in Fig. 15(a) in terms of the required OSNR to achieve BER $=10^{-3}$ versus

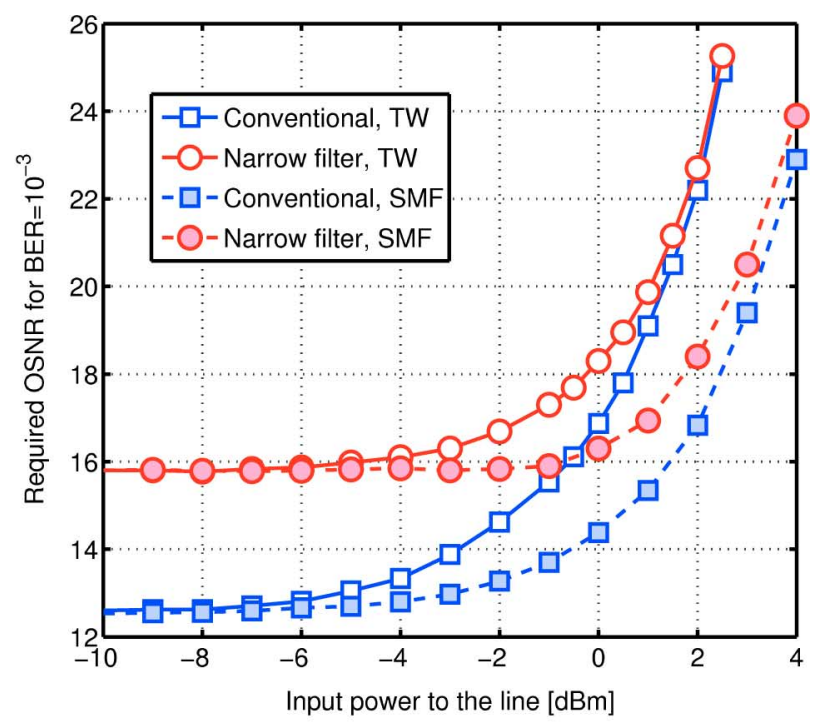

(a)

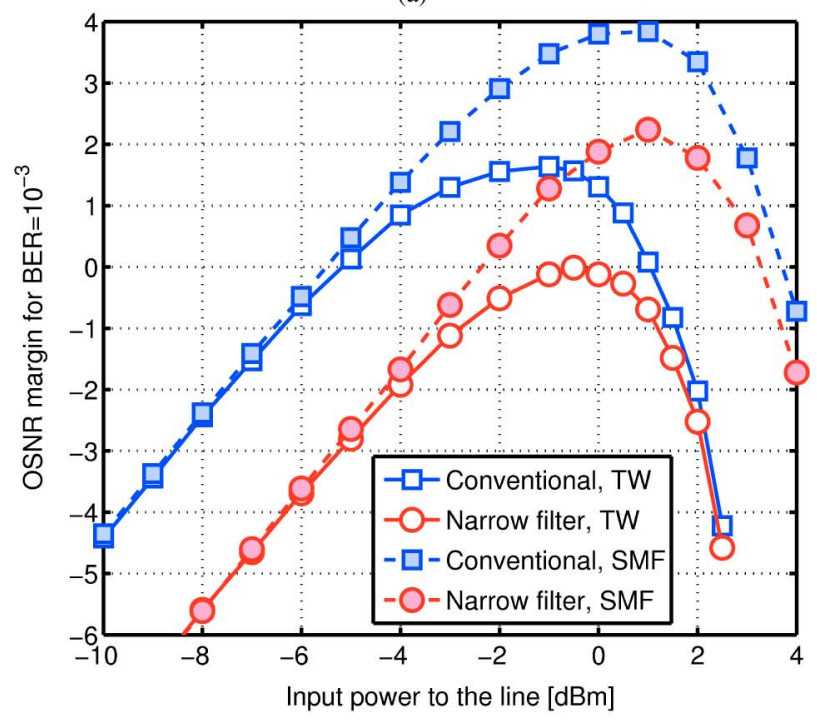

(b)

Fig. 15. Simulated tolerance to fiber nonlinearities. Fully compensated DQPSK 21 Gbaud single-channel $25 \times 80 \mathrm{~km}$ system.

signal input power (at BER $=10^{-5}$ such error-counting MC simulations are inpractical).

We observe that the $3 \mathrm{~dB}$ advantage of the conventional receiver is confirmed in the linear regime (low input power), but it diminishes as nonlinearities become the dominant source of errors. At large launch powers the performance difference between the two receivers is negligible both for TW and for SMF, confirming the conclusion we drew from our experimental setup. In Fig. 15(b), we report the OSNR margin for a $\mathrm{BER}=10^{-3}$, i.e., the difference between the actual OSNR of the line at $F=6 \mathrm{~dB}$, and the OSNR required for BER $=10^{-3}$. The optimum launch power achieving the largest OSNR margin [i.e., the nonlinear threshold (NLT)] is higher for SMF than for TW due to the larger local dispersion of SMF (NLT of $+0.5 \mathrm{dBm}$ instead of $-1.5 \mathrm{dBm}$ for conventional receiver, and of $+1 \mathrm{dBm}$ instead of $-0.5 \mathrm{dBm}$ in narrow filter receiver), but the difference in OSNR margin at the NLT between the $\mathrm{NF}$ receiver and the conventional receiver is no larger than approximately $1.6 \mathrm{~dB}$ for both SMF and TW fiber. 


\section{CONCLUSION}

We investigated a simplified receiver for DQPSK signals based on a single optical filter slightly offset from the carrier frequency, with 3-dB bandwidth smaller than the symbol rate. We have compared it to the conventional DQPSK receiver with respect to the major optical fiber impairments. The filter is implemented with one of the 32 channels of a standard AWG, a mature technology naturally enabling simultaneous demodulation of wavelength-division multiplexed DQPSK signals. We have reported experiments at $42 \mathrm{~Gb} / \mathrm{s}$, showing $\tilde{3}$ $\mathrm{dB}$ sensitivity penalty with respect to the conventional receiver in the linear regime. We have also shown that the narrow filter receiver has a stronger tolerance to accumulated $\mathrm{CD}$. We have compared simulation results against the experimental data, finding good agreement. Finally, we have discussed the impact of fiber nonlinearities on the performance of the receiver. We have shown in experiments and simulations that the advantage of the conventional receiver over the NF receiver diminishes when the fiber nonlinearities are taken into account.

\section{ACKNOWLEDGMENT}

The authors thank CMC Microsystems for providing access to the test equipment for these experiments, and P. Greig for the valuable experimental support.

\section{REFERENCES}

[1] P. J. Winzer and R.-J. Essiambre, "Advanced optical modulation formats," Proc. IEEE, vol. 94, no. 5, pp. 952-985, May 2006.

[2] P. J. Winzer, G. Raybon, H. Song, A. Adamiecki, S. Corteselli, A. H. Gnauck, D. A. Fishman, C. R. Doerr, S. Chandrasekhar, L. L. Buhl, T. J. Xia, G. Wellbrock, W. Lee, B. Basch, T. Kawanishi, K. Higuma, and Y. Painchaud, "100-Gb/s DQPSK transmission: From laboratory experiments to field trials," J. Lightw. Technol., vol. 26, no. 20, pp. 3388-3402, Oct. 2008.

[3] E. Ciaramella, G. Contestabile, and A. D'Errico, "A novel scheme to detect optical DPSK signals," IEEE Photon. Technol. Lett., vol. 16, no. 9, pp. 2138-2140, Sep. 2004.

[4] C. W. Chow and H. K. Tsang, "Polarization-independent DPSK demodulation using a birefringent fiber loop," IEEE Photon. Technol. Lett., vol. 17, no. 6, pp. 1313-1315, Jun. 2005.

[5] L. Christen, Y. Lize, S. Nuccio, L. Paraschis, and A. Willner, "Experimental demonstration of reduced complexity $43-\mathrm{Gb} / \mathrm{s}$ RZ-DQPSK rate-tunable receiver," IEEE Photon. Technol. Lett., vol. 20, no. 13, pp. 1166-1168, Jul. 2008.

[6] P. J. Winzer, S. Chandrasekhar, and H. Kim, "Impact of filtering on RZ-DPSK reception," IEEE Photon. Technol. Lett., vol. 15, no. 6, pp. 840-842, Jun. 2003.

[7] D. Penninckx, H. Bissessur, P. Brindel, E. Gohin, and F. Bakhti, "Optical differential phase shift keying (DPSK) direct detection considered as a duobinary signal," in Proc. 27th Eur. Conf. Opt. Commun. (ECOC 2001), , vol. 3, pp. 456-457.

[8] I. Lyubomirsky and C.-C. Chien, "DPSK demodulator based on optical discriminator filter," IEEE Photon. Technol. Lett., vol. 17, no. 2, pp. 492-494, Feb. 2005.

[9] A. D'Errico, R. Proietti, L. Giorgi, G. Contestabile, and E. Ciaramella, "WDM-DPSK detection by means of frequency-periodic Gaussian filtering," Electron. Lett., vol. 42, no. 2, pp. 112-114, 2006.

[10] E. Forestieri and G. Prati, "Narrow filtered DPSK implements order-1 CAPS optical line coding," IEEE Photon. Technol. Lett., vol. 16, no. 2 , pp. 662-664, Feb. 2004.

[11] L. Christen, Y. K. Lize, S. Nuccio, J.-Y. Yang, P. Saghari, A. E. Willner, and L. Paraschis, "Fiber Bragg grating balanced DPSK demodulation," in Proc. 19th Annu. Meeting IEEE Lasers Electro-Opt. Soc. (LEOS 2006), , pp. 563-564.
[12] G. Contestabile, R. Proietti, N. Calabretta, M. Presi, A. D'Errico, and E. Ciaramella, "Simultaneous demodulation and clock-recovery of 40-Gb/s NRZ-DPSK signals using a multiwavelength Gaussian filter," IEEE Photon. Technol. Lett., vol. 20, no. 10, pp. 791-793, May 2008.

[13] I. Lyubomirsky, C. Chien, and Y. Wang, "Optical DQPSK receiver with enhanced dispersion tolerance," IEEE Photon. Technol. Lett., vol. 20, no. 7, pp. 511-513, Apr. 2008.

[14] B. Mikkelsen, C. Rasmussen, P. Mamyshev, and F. Liu, "Partial DPSK with excellent filter tolerance and OSNR sensitivity," Electron. Lett., vol. 42, no. 23, pp. 1363-1364, 2006.

[15] C. Malouin, J. Bennike, and T. J. Schmidt, "Differential phase-shift keying receiver design applied to strong optical filtering," J. Lightw. Technol., vol. 25, no. 11, pp. 3536-3542, Nov. 2007.

[16] M. Haris, J. Yu, and G. Chang, "Impact of free spectral range optimization on RZ/NRZ DQPSK modulation format with strong optical filtering for ultrahigh data rate systems," in Proc. 20th Annu. Meeting IEEE Lasers Electro-Opt. Soc. (LEOS 2007), , pp. 141-142.

[17] Y. K. Lize, X. Wu, M. Nazarathy, Y. Atzmon, L. Christen, S. Nuccio, M. Faucher, N. Godbout, and A. E. Willner, "Chromatic dispersion tolerance in optimized NRZ-, RZ-, and CSRZ-DPSK demodulation," Opt. Exp., vol. 16, no. 6, pp. 4228-4236, 2008.

[18] V. Mikhailov, R. I. Killey, and P. Bayvel, "Experimental investigation of partial demodulation of $85.3 \mathrm{gb} / \mathrm{s}$ DQPSK signals," in Proc. 34th Eur. Conf. Opt. Commun. (ECOC 2008), pp. 1-2.

[19] H. Kim and A. H. Gnauck, "Experimental investigation of the performance limitation of DPSK systems due to nonlinear phase noise," IEEE Photon. Technol. Lett., vol. 15, no. 2, pp. 320-322, Feb. 2003.

[20] Y. Yadin, M. Orenstein, and M. Shtaif, "Balanced versus single-ended detection of DPSK: Degraded advantage due to fiber nonlinearities," IEEE Photon. Technol. Lett., vol. 19, no. 3, pp. 164-166, Feb. 2007.

[21] R. A. Griffin, "Integrated DQPSK transmitters," in Proc. Opt. Fiber Commun. Conf. 2005, , vol. 3, p. 3.

[22] S. Walklin and J. Conradi, "Effect of Mach-Zehnder modulator DC extinction ratio on residual chirp-induced dispersion in $10-\mathrm{Gb} / \mathrm{s}$ binary and AM-PSK duobinary lightwave systems," IEEE Photon. Technol. Lett., vol. 9, no. 10, pp. 1400-1402, Oct. 1997.

[23] H. Kim and A. Gnauck, "Chirp characteristics of dual-drive Mach-Zehnder modulator with a finite DC extinction ratio," IEEE Photon. Technol. Lett., vol. 14, no. 3, pp. 298-300, Mar. 2002.

[24] D. van den Borne, G. Khoe, H. de Waardt, and E. Gottwald, "Bit pattern dependence in optical DQPSK modulation," Electron. Lett., vol. 43, no. 22, 2007

[25] J.-S. Lee and C.-S. Shim, "Bit-error-rate analysis of optically preamplified receivers using an eigenfunction expansion method in optical frequency domain," J. Lightw. Technol., vol. 12, no. 7, pp. 1224-1229, Jul. 1994

[26] E. Forestieri, "Evaluating the error probability in lightwave systems with chromatic dispersion, arbitrary pulse shape and pre- and postdetection filtering," J. Lightw. Technol., vol. 18, no. 11, pp. 1493-1503, Nov. 2000.

[27] E. Forestieri, "(Corrections to) evaluating the error probability in lightwave systems with chromatic dispersion, arbitrary pulse shape and preand postdetection filtering," J. Lightw. Technol., vol. 21, no. 6, p. 1592, Jun. 2003.

[28] G. Bosco, A. Carena, V. Curri, R. Gaudino, P. Poggiolini, and S. Benedetto, "A novel analytical approach to the evaluation of the impact of fiber parametric gain on the bit error rate," IEEE Trans. Commun., vol. 49, no. 12, pp. 2154-2163, Dec. 2001.

[29] J. Wang and J. M. Kahn, "Impact of chromatic and polarization-mode dispersions on DPSK systems using interferometric demodulation and direct detection," J. Lightw. Technol., vol. 22, no. 2, pp. 362-371, Feb. 2004.

[30] P. Serena, A. Orlandini, and A. Bononi, "Parametric-gain approach to the analysis of single-channel DPSK/DQPSK systems with nonlinear phase noise," J. Lightw. Technol., vol. 24, no. 5, pp. 2026-2037, May 2006.

[31] H. Kim and P. Winzer, "Robustness to laser frequency offset in directdetection DPSK and DQPSK systems," J. Lightw. Technol., vol. 21, no. 9, pp. 1887-1891, Sep. 2003.

[32] K. Ho, "The effect of interferometer phase error on direct-detection DPSK and DQPSK signals," IEEE Photon. Technol. Lett., vol. 16, no. 1, pp. 308-310, Jan. 2004.

[33] G. Bosco and P. Poggiolini, "On the joint effect of receiver impairments on direct-detection DQPSK systems," J. Lightw. Technol., vol. 24, no. 3, pp. 1323-1333, Mar. 2006. 
[34] A. H. Gnauck, P. J. Winzer, C. Dorrer, and S. Chandrasekhar, "Linear and nonlinear performance of $42.7-\mathrm{Gb} / \mathrm{s}$ single-polarization RZ-DQPSK format," IEEE Photon. Technol. Lett., vol. 18, no. 7, pp. 883-885, Apr. 2006.

[35] J. P. Gordon and L. F. Mollenauer, "Phase noise in photonic communications systems using linear amplifiers," Opt. Lett., vol. 15, pp. 1351-1353, 1990.

[36] M. C. Jeruchim, P. Balaban, and K. S. Shanmugan, Simulation of Communication Systems: Modeling, Methodology, and Techniques. New York: Springer-Verlag, 2000.

[37] G. P. Agrawal, Nonlinear Fiber Optics, 3rd ed. New York: Academic, 2001.

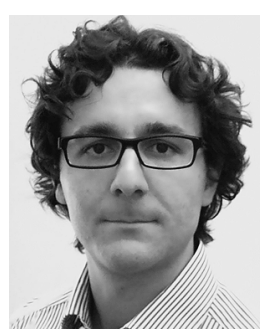

Francesco Vacondio was born on December 18, 1981 in Reggio Emilia, Italy. He received the Laurea Magistrale degree (cum laude) in electrical engineering from the University of Parma, Parma, Italy. He is working toward the Ph.D. degree in the Department of Electrical and Computer Engineering and the Center for Optics, Photonics and Lasers (COPL) at Université Laval, Québec, QC, Canada.

His research interests include performance analysis and design of spectrally efficient optical networks, the exploitation of nonlinear properties of semiconductor optical amplifiers, and microwave photonics.

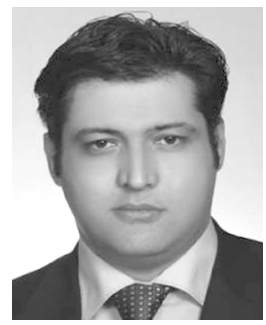

Amirhossein Ghazisaeidi received the M.A. degree in communications systems and the B.S. degree in electrical engineering from Sharif University of Technology, Tehran, Iran. Currently, he is working toward the Ph.D. degree at the Department of Electrical and Computer Engineering, Laval University, QC, Canada.

His recent research interests include optical-codedivision multiple access and spectrum-sliced WDM using incoherent sources, dynamics, and noise properties of optical amplifiers, modeling optoelectronic devices, and performance analysis of optical links.

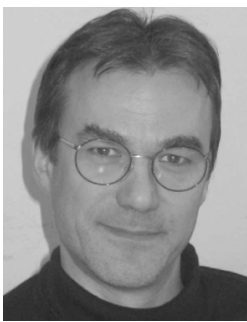

Alberto Bononi (M'90) received the Laurea degree (cum laude) in electronics engineering from the University of Pisa, Pisa, Italy, in 1988, and the M.A. and $\mathrm{Ph} . \mathrm{D}$. degrees in electrical engineering from Princeton University, Princeton, NJ, 1992, and 1994, respectively.

In 1990, he worked at GEC-Marconi Hirst Research Centre, on a Marconi S.p.A. project on coherent optical systems. From 1994 to 1996, he was an Assistant Professor in the Electrical and Computer Engineering Department, State University of New York (SUNY), teaching courses in electric circuits and optical networks. In the summers of 1997 and 1999, he was a Visiting Faculty at the Département de Genie Électrique, Université Laval, doing research on fiber amplifiers. Currently, he is an Associate Professor of telecommunications at the School of Engineering, Università di Parma, Parma, Italy, where he teaches courses in probability theory and stochastic processes, telecommunications networks, and optical communications. His recent research interests include system design and performance analysis of high-speed all-optical networks, nonlinear fiber transmission for WDM systems, linear and nonlinear polarization-mode dispersion, and transient gain dynamics in optical amplifiers.

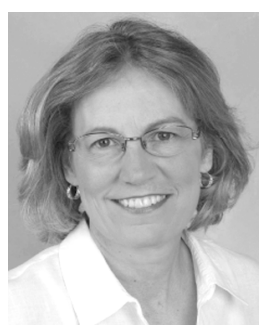

Leslie Ann Rusch (S'91-M'94-SM'00) received the B.S.E.E. degree (with honors) from the California Institute of Technology, Pasadena, in 1980, and the M.A. and Ph.D. degrees in electrical engineering from Princeton University, Princeton, NJ, in 1992 and 1994, respectively.

She spent two years as the Manager of a group researching new wireless technologies at Intel Corp. from 2001 to 2002. In 1994, she joined the Department of Electrical and Computer Engineering, Université Laval, QC, Canada, where she is currently a Full Professor performing research in wireless and optical communications. Her recent research interests include optical-code-division multiple access and spectrum-sliced WDM using incoherent sources for passive optical networks, semiconductor and erbium-doped optical amplifiers and their dynamics, radio over fiber, and in wireless communications, high-performance, reduced complexity receivers for ultra-wide-band systems employing optical processing. 